\title{
Sexually transmitted diseases in rape victims
}

\author{
S Estreich, G E Forster, A Robinson
}

\begin{abstract}
From 1 January 1986 to 1 September 1989124 women presented to the Ambrose King Centre (the department of genitourinary medicine of the London Hospital) alleging rape. Sexually transmitted diseases were found in $36(29 \%)$ women (excluding candidosis and bacterial vaginosis). The commonest organisms detected were Neisseria gonorrhoeae and Trichomonas vaginalis, each being present in 15 patients. Eleven women had genital warts. Chlamydia trachomatis was isolated in six patients, two had herpes simplex virus infection and one patient had pediculosis pubis. Serological evidence of past hepatitis $B$ infection was detected in five women and one patient had antibodies to human immunodeficiency virus. Eighteen of the 36 women $(50 \%)$ had multiple infections. Six women had abnormal cervical cytology smears, three being suggestive of cervical intraepithelial neoplasia grades II-III. Although it is rarely possible to attribute infection to an assailant, these patients require further counselling, treatment and review. Rape victims are thus a population at risk of having sexually transmitted diseases and screening should be offered.
\end{abstract}

\begin{abstract}
Introduction
The Sexual Offences Act (Amendment), 1976, in the UK defines rape as sexual intercourse with a woman without her consent, either by using force or fraudulent means. ${ }^{1}$ Vaginal penetration to any degree is sufficient. The risk of acquisition of sexually transmitted diseases (STDs) after sexual assault is unknown. A recent review ${ }^{2}$ highlighted the lack of data on the association between rape and STD, particularly conditions other than the traditional venereal diseases gonorrhoea and syphilis. Much of the available information has come from the microbiological assessment of women referred by police agencies in North America ${ }^{3-5}$ where the legal definitions of rape and child sexual abuse differ from
\end{abstract}

The Ambrose King Centre, The Royal London Hospital, London E1 1BB, UK

S Estreich, G E Forster, A Robinson those in the UK and where prophylactic treatment is more readily available. These studies have not always differentiated children from adults, girls aged less than 16 years representing up to $42 \%$ of victims in one study. ${ }^{6}$ More recently, infection with Chlamydia trachomatis has also been reported following rape. ${ }^{7}$

The viral STDs, namely human papilloma virus (HPV), herpes simplex virus (HSV), cytomegalovirus (CMV), hepatitis B virus (HBV), human immunodeficiency virus (HIV) and molluscum contagiosum may be acquired through rape. However, only two studies and one case report have considered the risk of acquisition of these infections. In a study ${ }^{7}$ of 46 women one had a four week history of genital warts, having attended 12 months after rape. No voluntary sexual activity had occurred between the assault and her initial visit. HSV infection was not reported in these patients and none were tested for antibodies to HIV. In a more recent study, 204 women were reviewed within 72 hours of rape by an unknown assailant or assailants. No cases of genital warts were reported. However, 13 of 199 women $\left(7^{\circ}\right)$ had evidence of koilocytosis on cervical cytology at their initial visit. HSV was isolated in four of 170 women $\left(2.4^{\circ}\right)$ at presentation. One of 123 patients $\left(0.8^{\circ}{ }_{0}\right)$ had antibodies to HIV detected at her first attendance. No additional viral infections occurred in 52 patients $(26 \%)$ during follow up ranging from two to 40 weeks, with a mean of eight weeks. The authors concluded that viral STD were not likely to be acquired as a consequence of sexual assault. Screening for markers of $\mathrm{HBV}$ infection was not carried out in either study. In addition, seroconversion to HIV has been reported ${ }^{9}$ in one woman within three months of a rape, where no other identifiable risk factors were present.

The difficulties in diagnosis and management of patients with viral STD have increased the workload of departments of genitourinary medicine in England. ${ }^{10}$ They require extensive counselling and follow up which may need to be intensified if the infections are diagnosed after rape. There is no simple confirmatory test for the clinical diagnosis of genital warts and the serological diagnoses of HBV and HIV infections may be associated with a lag period before a detectable response. The duration of infection is often impossible to establish particularly in relation to the timing of a rape. Genital warts may have a lengthy incubation period of up to nine months. HSV infection may be inapparent initially 
and remain latent until reactivation. Duration of infection may sometimes be estimated by documenting seroconversion in HSV, HBV and HIV infection.

The management of established infection with the viral STDs is unsatisfactory. The associated long term complications of these infections are an additional burden on the rape victim. HPV is associated with ano-genital neoplasia, ${ }^{11}$ HBV may result in chronic liver disease and carcinoma and HIV is complicated with life threatening opportunistic infections and tumours.

The objective of this study was to assess the overall frequency of STDs in rape victims who presented to a department of genitourinary medicine.

\section{Methods}

Between 1 January 1986 and 1 September 1989 all women attending the Ambrose King Centre, the department of genitourinary medicine at the London Hospital alleging rape were assessed according to a standardised protocol. A detailed history of the incident was taken, where appropriate, together with a sexual and medical history. Signs of assault and infection were documented at examination.

Material from the posterior fornix of the vagina was Gram-stained for candidal pseudohyphae/ spores and "clue cells" as well as suspended in saline and examined microscopically for Trichomonas vaginalis. Candida species were also cultured from the vaginal material in Sabouraud's medium and $T$. vaginalis infection was confirmed by acridine orange staining. Bacterial vaginosis was diagnosed by the presence of "clue cells", positive amine test and a vaginal $\mathrm{pH}$ of greater than 5.5. Gram-stained smears from the urethra, endocervix and rectum were examined for Gram-negative intracellular diplococci. Material from the urethra, endocervix and rectum and, if appropriate, the pharynx, were directly plated on to brain heart infusion agar for culture for Neisseria gonorrhoeae. Isolates were identified on sugar solid media. Material from the same sites were placed in 2-sucrose phosphate media, and transported in liquid nitrogen to the laboratory for culture for $C$. trachomatis. Cervical cytological smears were performed in all the women. Genital warts were clinically diagnosed. Cultures for herpes simplex virus were taken as clinically indicated. Specimens were transported in Virocult transport media (Medical Wire and Equipment Co Ltd) and cultured in human embryonic lung cells. Evidence of cytopathic effect was noted at weekly intervals for three weeks.

A bimanual pelvic examination was performed. Serological tests for syphilis and HBV were carried out at the initial assessment. HBV serology was performed using the Wellcozyme method. Serum for antibodies to HIV was tested by ELISA methods and
Table 1 Referral patterns of rape victims

\begin{tabular}{lc}
\hline Referral agency & $\begin{array}{l}\text { Number of } \\
\text { patients }\left({ }^{\circ}\right)\end{array}$ \\
\hline Self & $69(56)$ \\
General practitioner & $29(23)$ \\
Police & $20(16)$ \\
Voluntary agencies & $6(5)$ \\
Total & $124(100)$ \\
\hline
\end{tabular}

confirmed using gel particle agglutination. An additional serum sample was saved for possible paired testing at a later date. Tests for antibodies to HIV were taken only after counselling and informed consent.

Follow-up was arranged after two weeks and three months when Gram-stained smears and cultures for $N$. gonorrhoeae and $C$. trachomatis were repeated. Serological tests were also repeated at the last visit. A third visit was not arranged if the initial assessment was three months or more from the rape. Counselling was initially provided by the clinic health advisers who also informed the patients of other support agencies and made referrals, where appropriate.

\section{Results}

\section{Epidemiology}

Of 141 women attending the Ambrose King Centre from 1 January 1986 to 1 September 1989 following sexual assault, 124 women (mean age 26 years, range 16-45 years) alleged that rape had occurred. This took place in Britain in 110 cases $(89 \%)$, Mediterranean countries in eight $(6.5 \%)$, Africa in four $\left(3^{\circ}{ }_{0}\right)$ and North America in two (2\%). One hundred and five victims ( $85 \%$ ) were Caucasian, 14 $\left(11^{\circ}{ }_{0}\right)$ were Afro-Caribbean and four $(3 \%)$ were from other ethnic backgrounds. Fifty seven $(46 \%)$ presented within two weeks of the assault (range of one day to six years). The number of clinic visits made by the patients ranged from one to seven. After the initial visit, 104 women ( $84 \%$ ) attended for a first follow-up at a mean of 31 days (range 3 days to 96 weeks) and $76(61 \%)$ attended for a second review at a mean of 11 weeks (range 1-100).

Most of the women, $69(56 \%)$, were self-referred. Other sources of referral are shown in table 1 . The rape had been reported to the police by 58 women

Table 2 Epidemiological features of the rape

\begin{tabular}{ll}
\hline Feature & $\begin{array}{l}\text { Number of } \\
\text { patients }(\%) \\
(n=124)\end{array}$ \\
\hline No prior sexual experience & $7(6)$ \\
Known assailant & $55(44)$ \\
Oral penetration & $14(11)$ \\
Voluntary intercourse after rape & $50(40)$ \\
\hline
\end{tabular}

*In addition to vaginal penetration. 
$(47 \%)$. Further epidemiological features of the assault are shown in table 2 . The rape was their first sexual experience for seven women. Fifty $(43 \%)$ of 117 women with prior sexual experience had voluntary sexual intercourse with another partner after the assault. A past history of rape was given by four women $(3 \%)$ and child sexual abuse by three women $(2 \%)$. Twenty four women $(19 \%)$ had been raped by more than one assailant and $13(10 \%)$ had been subjected to anal penetration (in addition to vaginal penetration). Five women had evidence of vulval injury, all presenting within two weeks of the rape.

\section{Sexually transmitted infections}

The frequency of sexually transmissible infections is shown in table 3 . Overall, $36(29 \%)$ women had 57 separate STDs. Vaginal candidosis and bacterial vaginosis were diagnosed in 22 and seven women respectively but are excluded from these data. The commonest organisms detected were $N$. gonorrhoeae (no penicillinase producing organisms were isolated) and $T$. vaginalis, each being present in 15 patients. Genital warts were diagnosed in 11 women and C. trachomatis was found in six women. Of 123 women tested none had positive serological tests for syphilis. Clinical features of pelvic inflammatory disease were present in six women, of whom two had gonococcal cervicitis, one had trichomoniasis and one had bacterial vaginosis. No pharyngeal infections were associated with a history of oral penetration in 14 women. Anal penetration was associated with one case of rectal gonorrhoea. Of the seven women with no prior sexual experience, one had trichomoniasis and the other had candidal infection.

Of the two women with HSV infection one had a history of recurrent vulval disease and was seen four years after the rape. The timing of the primary attack was unknown. The other was seen eight days after being raped but had had symptoms for two weeks localised to the labia minora.

Antibodies to hepatitis B surface antigen (HBsAg) were detected in four women. Three were tested within two months of being raped, the fourth after six months. A fifth woman was both HBsAg positive and had antibodies to hepatitis $B$ e antigen, being tested 18 days after the rape. Three of these women were from countries of high $\mathrm{HBV}$ prevalence and two were ex-injecting drug users. No cases of acute seroconversion were reported during follow up. Of the 87 women who had negative HBV serology, 37 (43\%) were either initially tested or retested at three months or more.

The single patient (of 44 tested) with antibodies to HIV presented as a contact of her regular male partner who had gonorrhoea. She had been raped abroad two months previously. No oral or anal penetration had occurred. Samples from both her
Table 3 Overall frequency of sexually transmissible infections in victims of rape

\begin{tabular}{ll}
\hline $\begin{array}{l}\text { Sexually } \\
\text { transmissible } \\
\text { infections }\end{array}$ & $\begin{array}{l}\text { Number of } \\
\text { patients } \\
(n=124)\end{array}$ \\
\hline Gonorrhoea & $15)$ \\
Trichomoniasis & $15(12)$ \\
Genital warts & $11(8)$ \\
Chlamydial infection & $6(5)$ \\
Genital herpes & $2(2)$ \\
Pediculosis pubis & $1(1)$ \\
Hepatitis B markers & $5 / 92$ \\
HIV antibody & $1 / 44$ \\
\hline
\end{tabular}

* 18 patients with multiple infections.

initial and two month follow up visits showed antibodies to HIV. Her partner remained HIV antibody negative and she denied other sexual contacts or risk factors. Overall, $24(19 \%)$ patients expressed anxiety about HIV infection as a consequence of rape (table 4). In eight women it was the reason for their initial attendance. Seven of this group declined HIV testing after counselling. HIV tests were performed at a mean of eight months (range one month to four years) in 43 women where the result was subsequently negative. After pre-test counselling, 42 women $(34 \%)$ declined to proceed with the HIV test.

Eighteen of the 36 women (50\%) with an STD had multiple infections (table 5). Dual infections were present in 15 women and three had triple infections.

For $15(12 \%)$ women an STD was found on follow up which had not been detected at their initial assessment. Four of these women had not had further intercourse from the time of the rape to the detection of the infection. The first woman had chlamydial infection which was detected at three months and was not found at her initial visit three weeks from the rape. The second woman presented with pediculosis pubis at five months, this infestation not being detected at her initial visit five days from the rape. The other two women had genital warts. Of the 11 women who had had intervening sexual intercourse four had gonorrhoea, three had trichomoniasis, one had both and three had genital warts.

Cervical cytology

Of 108 evaluable cervical cytology smears six $(6 \%)$

Table 4 Women expressing HIV anxiety: HIV antibody tests

\begin{tabular}{lccr}
\hline $\begin{array}{l}\text { Year of } \\
\text { study }\end{array}$ & $\begin{array}{l}\text { Number of } \\
\text { women }\end{array}$ & $\begin{array}{l}\text { HIV anxiety } \\
\left({ }_{0}{ }_{0}\right)\end{array}$ & $\begin{array}{l}\text { HIV tests } \\
\left({ }_{0}\right)\end{array}$ \\
\hline 1986 & 21 & $3(14)$ & $8(38)$ \\
1987 & 31 & $6(19)$ & $14(45)$ \\
1988 & 44 & $12(27)$ & $18(41)$ \\
$1989 \star$ & 28 & $3(11)$ & $4(14)$ \\
Total & 124 & $24(19)$ & $44(36)$ \\
\hline
\end{tabular}

^Data up to 1 September 1989. 
Table 5 Multiple sexually transmitted diseases in rape victims

\begin{tabular}{lllll}
\hline $\begin{array}{l}\text { Sexually } \\
\text { transmitted } \\
\text { disease }\end{array}$ & $\begin{array}{l}\text { Genital } \\
\text { warts }\end{array}$ & $H B V$ & HIV & TV \\
\hline Chlamydial infection & $2^{\star}$ & 0 & 0 & 0 \\
Gonorrhoea & $2^{\star}$ & 0 & 0 & 8 \\
Herpes simplex & 1 & 0 & 0 & 0 \\
TV & 3 & 1 & $1 \dagger$ & - \\
Total & 8 & 1 & 1 & 8 \\
\hline
\end{tabular}

*One woman had concurrent TV.

†This patient also had antibodies to HBsAg.

were abnormal. Three smears showed changes suggestive of cervical intra-epithelial neoplasia (CIN), all of which were grades II or III. In two of these women colposcopic biopsy showed no evidence of dysplasia and the third was lost to follow up after referral to another hospital. Two cervical smears demonstrated koilocytic changes suggestive of HPV infection. Colposcopic biopsy was negative in one of the women who had clinical vulval warts and showed evidence of CIN II in the other woman who was referred for laser therapy. One smear showed inflammatory changes in association with $T$. vaginalis infection. This was negative on repeating after appropriate therapy.

\section{Antibiotic use}

Although antibiotics had been used by 10 women from two days to two months prior to their assessment, infections were detected in four of them. Two women had $N$. gonorrhoeae isolated having taken erythromycin and metronidazole, one woman had both $N$. gonorrhoeae and $T$. vaginalis detected having taken co-trimoxazole. The fourth had both genital warts and $T$. vaginalis detected having taken phenoxymethyl penicillin. Other antibiotics used were ceftriaxone, amoxycillin and oxytetracycline, all potentially active against some STDs.

\section{Contraception}

Seventy women $(56 \%)$ were not using any method of contraception with their regular partner at the time of the sexual assault (table 6). Condoms were not used by any of the assailants. Post-coital hormonal

Table 6 Contraceptive methods used by women in the study at the time of rape

\begin{tabular}{ll}
\hline $\begin{array}{l}\text { Method of } \\
\text { contraception }\end{array}$ & $\begin{array}{l}\text { Number of } \\
\text { patients } \%) \\
(n=124)\end{array}$ \\
\hline None & $70(56)$ \\
Combined oral contraceptive & $25(20)$ \\
Condom & $16(13)$ \\
Sterilisation & $6(5)$ \\
Intra-uterine contraceptive device & $5(4)$ \\
Diaphragm & $1(1)$ \\
Depo-provera & $1(1)$ \\
\hline
\end{tabular}

contraception was prescribed for 13 women. Three pregnancies were associated temporally with the rapes and in one case the woman had no current sexual partner. Two pregnancies were terminated, the third patient was not followed up after her third visit.

\section{Discussion}

STDs (excluding vaginal candidosis and bacterial vaginosis) were present in $36(29 \%)$ of 124 rape victims attending the Ambrose King Centre during the study period. The commonest organisms detected were $N$. gonorrhoeae and $T$. vaginalis, each being found in 15 women. Although C. trachomatis is one of the commonest STDs in the United Kingdom it was isolated in only six women. There are many variables in the isolation of $C$. trachomatis. Culture from a single swab from each site may be associated with a false negative result. ${ }^{12}$

No women were found to have reactive serological tests for syphilis. Previous studies have shown similar results. ${ }^{3-7}$ Syphilis is now an uncommon infection in this country, probably due to continued screening and contact tracing. These findings should reinforce current screening policies rather than lead to complacency. ${ }^{13}$

The most common viral infection was with HPV in the form of genital warts, being present in $11(9 \%)$ patients. In five women the warts were not detected at their initial visit but only on follow up. This suggests that the infection either did not pre-date the rape or that at the initial visit the patient had subclinical disease. Eight of this group had one or more coexistent STD. It is possible that cases of wart virus infection were missed because of the long incubation period and that additional cases may have been detected on extended review. The use of the colposcope may have increased the yield of HPV detection but has the disadvantage of being perceived as an additional trauma by the rape victim. Although DNA typing was not undertaken in this study it may be desirable in providing medico-legal evidence.

Two women had evidence of symptomatic HSV infection. In both cases these were unlikely to have been associated with the rape. However, inapparent HSV infection would not have been detected in this study since viral cultures were only performed on clinical grounds. HSV is often complicated by psychological morbidity. ${ }^{14}$ This problem could be exaggerated if the infection were acquired following rape.

No acute cases of $\mathrm{HBV}$ infection were detected. One low risk carrier of HBV was identified 18 days after the rape. Probably all of the women with HBV markers acquired their infection prior to their assault. This is the first study to present data on $\mathrm{HBV}$ in victims of rape. However, if the assailant is 
suspected of being an infective risk, post-exposure HBV prophylaxis should be considered. ${ }^{15}$

The increasing trend in the number of HIV tests performed from 1986 to 1988 shown in table 5 may have reflected publicity surrounding $\mathrm{HIV}$ at that time. Eight women attended solely because they perceived themselves to be at risk of HIV infection following rape, one decided not to be tested after counselling. Of the $24(19 \%)$ women expressing anxiety about AIDS, seven declined HIV testing after counselling.

Only one woman had antibodies to HIV and she did not present with HIV anxiety. It was not possible to directly attribute this to the rape since antibodies were present at the initial visit two months after the rape had occurred. The HIV status of her assailant was unknown. Supporting data were presented in Jenny's series from Seattle ${ }^{8}$ in which no seroconversions were recorded in 123 patients, of whom $52(42 \%)$ returned for a second follow up visit at a mean of eight weeks. A recent case report of HIV seroconversion after rape $^{9}$ has highlighted this potential risk for rape victims. This has prompted discussion about post-rape prophylaxis using zidovudine. ${ }^{9}$ As in the case of needle stick injury, ${ }^{16}$ no data are available on the efficacy of this treatment. The topical use of substances with anti-HIV activity, ${ }^{17}$ such as nonoxynol-9, following rape has been suggested but these products have not been evaluated, do not consider the possibility of multiple sites being involved and may even be harmful. ${ }^{18}$ Discussion about HIV with a woman in such a vulnerable state following rape may not be appropriate in all cases. ${ }^{19}$ Our practice is to save a serum sample at the initial visit to be tested when an informed decision can be made and repeated, as necessary. However, the risk of HIV transmission is small, at least in the low prevalence areas so far studied, but this may well change with time. The risk of HIV transmission may also be affected by the use of condoms by assailants, the occurrence of anal penetration, anogenital trauma and rapes by multiple assailants.

Three of six abnormal smears showed changes suggestive of significant $\mathrm{CIN}$ abnormalities. In view of the natural history of CIN and the relatively short time period to detection in this study it is unlikely that these abnormalities were related to the rape. However, they represent important incidental findings in need of assessment which may otherwise have remained undetected. ${ }^{20}$

The presence of an STD cannot necessarily be attributed to an assault. It is possible that the infection was present before the rape. This could be excluded in one patient with $T$. vaginalis infection who had been a virgin. An important confounding factor in linking an STD to an assailant is the occurrence of voluntary sexual intercourse between the rape and medical review, introducing the possibility of STD acquisition after the rape. Fifty $\left(43^{\circ}{ }_{0}\right)$ of 117 women with prior sexual experience had voluntary sexual intercourse with another partner after the rape. Of those women found to have an STD, five of their sexual partners were traced. All had corresponding infections. However, this does not elucidate the source of the STD. Despite the assailant being known to $55(44 \%)$ women we did not have the opportunity to screen any of them. If an alleged assailant does present for STD screening it would be prudent for medico-legal purposes to store specimens, in particular gonococcal isolates, which can be both serotyped and auxotyped, ${ }^{21}$ if necessary, at a reference laboratory. These specimens could be compared to stored isolates from the victim to either help corroborate or refute the link between assailant and victim. In a similar way, immunotyping of chlamydial isolates could be performed if facilities permit.

Results of medical investigations may be required as evidence in pending legal cases. Culture of $N$. gonorrhoeae and $C$. trachomatis can be used as firm evidence for the presence of infection. Chlamydial antigen detection methods using immunofluorescence or enzyme immune assay (EIA) are becoming more widely available. They are useful in clinical management for screening populations at risk of chlamydial infection. They should not be used in medico-legal evidence ${ }^{22}$ because of their lower specificity. However, EIA modified with blocking techniques has improved predictive values (Schachter et al, International Society for Sexually Transmitted Disease Research, Abstract no. 19, Copenhagen, 1989), but the problem of false positive results still exists.

Intercurrent antibiotics used by 10 women may have masked infection in the six women in whom no STD was detected. The antibiotics used were all potentially active against some STD.

When raped $36(29 \%)$ women had adequate protection against pregnancy (either using the combined oral contraceptive, an intra-uterine contraceptive device or had been sterilised). Of those with inadequate contraception three became pregnant and two had terminations. Post-coital hormonal contraception had been given to 13 women; it had not been prescribed for the three women who became pregnant because they all presented more than five weeks from the assault. In patients with chlamydial infection pelvic inflammatory disease may result from termination of pregnancy ${ }^{23}$ if these infections are not sought and treated.

The range of STD detected mitigates against the use of prophylactic antibiotic regimes following rape. Thorough investigation and treatment of identified infection is a more satisfactory alternative. ${ }^{22}$ However, epidemiological treatment may be 
appropriate if a patient cannot return for follow up and compound regimes have been suggested. ${ }^{22}$

Screening for STDs is only part of the overall management of rape victims. Psychological and emotional support should be made available. The patients in our study were offered immediate access to health advisers within the clinic for initial counselling. Further tiers of psychological support were provided by other statutory and voluntary agencies as appropriate.

In conclusion, we have shown that rape victims are a population at risk of having STDs. However, in only a minority of cases is it possible to attribute the STD to an assailant. Screening for STDs should now be offered in the management of rape victims. It may also be necessary to make provision for storing specimens for further analysis where medico-legal cases are likely.

We thank all the staff of the Ambrose King Centre for their help in the sympathetic management of the patients reported in this study. We also thank Dr J Treharne of the Institute of Ophthalmology, London, for performing chlamydial isolation.

\section{Address correspondence to Dr S Estreich.}

1 "Unlawful Sex". The Report of a Howard League Working Party. Waterlow Publishers Ltd, 1985:169-71.

2 Glaser JB, Hammerschlag MR, McCormack WM. Epidemiology of sexually transmitted diseases in rape victims. Rev Infect Dis 1989;11:246-54.

3 Hayman CR, Lanza C. Sexual assault on women and girls. Am J Obstet Gynecol 1971;109:480-6.

4 Hayman CR, Lanza C, Fuentes R, Algor K. Rape in the District of Columbia. Am J Obstet Gynecol 1972;113:91-7.
5 Everett RB, Jimerson GK. The rape victim: a review of 117 consecutive cases. Obstet Gynecol 1977;50:88-90.

6 Evrard JR, Gold EM. Epidemiology and management of sexual assault victims. Obstet Gynecol 1979;53:381-7.

7 Forster GE, Pritchard J, Munday PE, Goldmeier D. Incidence of sexually transmitted diseases in rape victims during 1984 . Genitourin Med 1986;62:267-9.

8 Jenny C, Hooton TM, Bowers A, et al. Sexually transmitted diseases in victims of rape. $N$ Engl J Med 1990;322:713-6.

9 Murphy S, Kitchen V, Harris JRW, Forster SM. Rape and subsequent seroconversion to HIV. Br Med J 1989;299:718.

10 Thin RNT. Workloads in genitourinary medicine clinics in England. Genitourin Med 1989;65:376-81.

11 Oriel JD. Sex and cervical cancer. Genitourin Med 1988;64:81-9.

12 Dunlop EMC, Goh BT, Darougar S, Woodland R. Triple culture tests for diagnosis of chlamydial infection of the female genital tract. Sex Transm Dis 1985;12:68-71.

13 Clay JC. Antenatal screening for syphilis. (Editorial). $\mathrm{Br} \mathrm{Med} \mathrm{J}$ 1989;299:409-10.

14 Goldmeier D, Johnson A, Jeffries D, et al. Psychological aspects of recurrences of genital herpes. J Psychosom Res 1986;30: 601-8.

15 Iwarson S. Post-exposure prophylaxis for hepatitis B: active or passive? Lancet 1989;ii:146-8.

16 Centers for Disease Control. Public health service statement on management of occupational exposure to HIV, including consideration regarding zidovudine postexposure use. MMWR 1990;39(no.RR-1).

17 Foster IM, Bartlett S. Anti-HIV substances for rape victims (letter). JAMA 1989;261:3407.

18 Murphy SM, Munday PE, Jeffries DJ. Anti-HIV substances for rape victims (letter). JAMA 1989;262:2090-1.

19 Forster GE, Pritchard J, Munday PE, Goldmeier D. Risk of AIDS after rape (letter). Genitourin Med 1987;63:217.

20 Seltzer VC, Hassman H, Bigelow B. Abnormal Papanicolaou smears found in victims of sexual assault. J Reprod Med 1978;20:233.

21 Catlin BW. Nutritional profiles of Neisseria gonorrhoeae, $N$. meningitides, $N$. lactamica in chemically defined media and the use of growth requirements for gonococcal typing. J Infect Dis 1973;128:178-94.

22 Centers for Disease Control. Sexual assault and STD. MMWR 1989;38:40-3.

23 Barbacci M, Spence MR, Kappus EW, et al. Post abortal endometritis and the isolation of Chlamydia trachomatis. Obstet Gynecol 1986;68:686-8.

Accepted for publication 15 August 1990 